\title{
Sequenz-Evaluation für die MR-Enterografie für Patienten mit Morbus Crohn
}

\author{
MR Enterography Sequence Evaluation for Patients with Crohn's Disease
}

\author{
Autoren \\ S. Schleder ${ }^{1}$, L.-M. Dendl' ${ }^{1}$, M. Pawlik² , C. Friedrich ${ }^{1}$, C. Ott ${ }^{3}$, A. Agha ${ }^{4}$, P. Wiggermann ${ }^{1}$, C. Stroszczynski ${ }^{1}$, \\ A. G. Schreyer ${ }^{1}$ \\ Institute \\ Institut für Röntgendiagnostik, Universitätsklinikum Regensburg \\ 2 Klinik für Anästhesiologie, Intensiv- und Notfallmedizin, Caritas-Krankenhaus St. Josef, Regensburg \\ 3 Klinik und Poliklinik für Innere Medizin I, Universitätsklinikum Regensburg \\ ${ }^{4}$ Klinik und Poliklinik für Chirurgie, Universitätsklinikum Regensburg
}

\author{
Key words \\ abdomen \\ - MR imaging \\ - efficacy studies
}

eingereicht 13.9 .2012

akzeptiert $\quad 12.12 .2012$

Bibliografie

DOI http://dx.doi.org/

$10.1055 / \mathrm{s}-0032-1330516$

Online-Publikation: 25.2.2013

Fortschr Röntgenstr 2013; 185 :

440-445 @ Georg Thieme

Verlag KG Stuttgart · New York . ISSN 1438-9029

\section{Korrespondenzadresse} Dr. Stephan Schleder

Institut für Röntgendiagnostik, Universitätsklinikum

Regensburg

Franz-Josef-Strauss-Allee 11

93053 Regensburg

Stephan.Schleder@ukr.de

Tel.: ++ 49/941/9447401

Fax: ++ 49/941/9447402

\section{Zusammenfassung}

$\nabla$

Ziel: Evaluation eines Routine-MRT-Protokolls für die MR-Enterografie (MRE) zur Untersuchung von Patienten mit Morbus Crohn (MC), um ein subjektives Ranking bezüglich wichtiger und weniger wichtiger Sequenzen zur Entscheidungsfindung hinsichtlich einer Diagnose zu erhalten.

Material und Methoden: 84 Patienten (42 männlich) mit histologisch gesichertem MC wurden prospektiv mit einer koronaren T2/T1-gewichtetenSequenz (TrueFISP), einer axialen T2-gewichteten Turbo-Spin-Echo-Sequenz (HASTE), einer axialen T1-gewichteten Gradienten-Echo-Sequenz (2DFLASH) sowie axialen und koronaren kontrastmittelverstärkten 2D-FLASH-Sequenzen untersucht. Die Sequenzen wurden von 4 erfahrenen Radiologen unabhängig voneinander subjektiv mit einer Schulnotenskala ( 1 = sehr gut; 5 = unbrauchbar) bezüglich ihrer Wichtigkeit in der Diagnosefindung und Bildbeurteilung benotet. Die sich aus der Benotung ergebende Rangfolge der MRT-Sequenzen wurde mit der Rangvarianz-Analyse nach Friedman auf ihre Signifikanz überprüft.

Ergebnisse: Es ergibt sich folgendes Ranking: HASTE vor kontrastmittelverstärkter 2D-FLASH koronar, vor TrueFISP und vor der kontrastmittelverstärkten 2D-FLASH axial, zuletzt die 2D-FLASH nativ. Der Unterschied zwischen der erstplatzierten HASTE und der zweitplatzierten kontrastmittelverstärkten 2DFLASH-Sequenz koronar war signifikant $(p<0,05)$, die weiteren Ergebnisse $(p<0,01)$ hochsignifikant. Schlussfolgerung: Die nativen, stabilen und schnell durchführbaren MRT-Sequenzen einer koronaren TrueFISP und einer axialen HASTE wurden subjektiv als Platz 1 und 3 im Ranking eingestuft. Das Untersuchungsprotokoll bei der MRE bei MC-Patienten sollte mit kontrastgestützten T1-gewichteten GradientenEcho-Sequenzen vor allem in koronarer Schichtführung ergänzt werden, ggf. mit einer Erweiterung auch in axialer Orientierung, wobei auf native T1-gewichtete Sequenzen verzichtet werden kann.

\section{Abstract \\ $\nabla$}

Purpose: To evaluate a routine MR enterography (MRE) protocol for patients with Crohn's disease (CD) in order to assess and rank the subjectively most important sequences regarding diagnostic decisions.

Materials and Methods: We prospectively examined 84 patients ( 42 male) with known $\mathrm{CD}$ using a coronal T2 / T1-weighted balanced SSFP (TrueFISP), axial T2-weighted single shot TSE (HASTE) as well as an axial T1-weighted gradient-echo sequence (2D-FLASH) before intravenous contrast application and a 2D-FLASH sequence with axial and coronal orientation after intravenous contrast application. 4 experienced radiologists subjectively evaluated the sequences independently using a scale between 1 and 5 ( $1=$ excellent; $5=$ non-diagnostic) regarding their diagnostic significance for a final radiologic decision. The ranking of the different sequences was statistically tested by the Friedman analysis.

Results: The following ranking was found: HASTE sequences were ranked prior to contrast-enhanced axial gradient-echo (2D-FLASH). The third to fifth ranking was TrueFISP, the axial contrast-enhanced 2D-FLASH and the 2D-FLASH without contrast, respectively. Differences between the first and second rank were significant $(\mathrm{p}<0.05)$, and all other differences were highly significant $(\mathrm{p}<0.01)$.

Conclusion: The stable and fast T2-weighted MR sequences without intravenous contrast represented by axial HASTE and coronal TrueFISP were ranked as number 1 and 3 . The examination protocol should be completed by a coronal T1weighted gradient-echo-sequence after contrast injection, which can be supplemented by an axial acquisition. The T1-weighted gradient-echo sequence without contrast could be omitted. 


\section{Einleitung}

\section{$\nabla$}

Die wesentlichen radiologischen bildgebenden Verfahren zur Primär- oder Rezidivdiagnostik bei Patienten mit Morbus Crohn (MC) sind neben der konventionell-radiologischen Doppelkontrastuntersuchung die abdominelle Sonografie mit Ultraschall (US), die Computertomografie (CT) und die Magnetresonanztomografie (MRT) [1]. Alle genannten Untersuchungsmethoden weisen Vor- und Nachteile auf, weswegen in der aktualisierten deutschen S3-Leitlinie zur Diagnostik des MC festgehalten wurde, dass nicht eine einzelne Untersuchung den Goldstandard darstellen kann [2, 3]. Auch die aktuellen europäischen Leitlinien beschreiben die Bedeutung aller Verfahren mit Priorisierung der MRT [4].

Die MRT bietet unter Einbeziehung der aktuellen technischen Entwicklungen den Vorteil, eine bildgebende Untersuchungsmethode mit hoher örtlicher und zeitlicher Auflösung zu sein und dabei ohne Strahlenexposition auszukommen. Sie wird daher in den Leitlinien der Deutschen Gesellschaft für Verdauungs- und Stoffwechselkrankheiten (DGVS) neben dem US als die favorisierte radiologische Basisdiagnostik des MC angesehen.

Die MRT des Dünndarms mit oraler Kontrastierung, die in der Literatur als MR-Enterografie (MRE) bezeichnet wird, repräsentiert eine in vielen Studien etablierte und wenig belastende Methode, die relevante Pathologien bei Patienten mit MC mit hoher Sensitivität und Spezifität detektieren kann [5, 6]. Zusätzlich können Aussagen über den Aktivitätsgrad der Erkrankung sowie eventuell vorliegender extraluminaler Komplikationen, wie etwa Abszesse oder Fisteln, getroffen werden, die endoskopischen Verfahren oder konventionell-radiologischen Doppelkontrastverfahren verborgen bleiben können. Die MRE zeigt auch eine exzellente Übereinstimmung mit aktuellen klinischen Score-Systemen wie etwa der Montreal-Klassifikation bezüglich der Beurteilung der Erkrankungsausprägung hinsichtlich Entzündungen, Stenosen oder perforierenden Prozessen [7].

Auch in aktuellen Metaanalysen erweist sich die MRE als eine der CT oder den konventionellen Verfahren gleichgestellte oder überlegene Methode [8].

Der MRT kommt in nahezu allen klinischen Fachbereichen eine steigende Bedeutung zu. Bei gleichzeitig hohen Untersuchungskosten durch personal- und investitionsintensive Fixkosten ist aus ökonomischen Gründen die Untersuchungszeit pro Untersuchungsgang ein wertvolles Gut [9]. Bei der aktuell bereits klinisch hohen Akzeptanz der MRE für die Evaluation von Patienten mit MC und gleichzeitig weiter bestehender Knappheit von MRT-Geräten kommt der Untersuchungszeit jeder Einzeluntersuchung eine zunehmende Bedeutung zu. Ziel dieser prospektiven Studie war es daher, ein optimales MRT-Sequenz-Protokoll für die Untersuchung von Patienten mit MC herauszuarbeiten um künftig ggf. verkürzte Sequenzalgorithmen zu etablieren.

\section{Material und Methode}

\section{Patientenkollektiv}

In die vorliegenden Studie wurden konsekutiv 84 Patienten (42 weiblich, 42 männlich) prospektiv eingeschlossen, welche bei einem histologisch gesicherten MC zu einer MRE angemeldet wurden. Bei keinem der angemeldeten Patienten bestand eine Kontraindikation zur Durchführung einer MRE. Das Alter zum Untersuchungszeitpunkt betrug zwischen 16 und 81 Jahren (Median 37 Jahre). Die MRE erfolgte im Rahmen der Erstdiagnostik oder zu Verlaufskontrollen bei neu aufgetretenen klinischen Symptomen.

\section{MR-Untersuchungsablauf}

Alle Patienten waren zum Untersuchungszeitpunkt mindestens 12 h nüchtern. Eine besondere Darmvorbereitung, z. B. Abführen, war für diese Untersuchungsmethode nicht notwendig. Um ein hypointenses intraluminales Signal in der T1-gewichteten Sequenz zu erzeugen („dark lumen Technik“), tranken die Patienten zunächst innerhalb $1 \mathrm{~h} 21$ Wasser mit zusätzlich $25 \mathrm{~g}$ Mannitol und $5 \mathrm{~g}$ Johannisbrotkernmehl (Nestargel, Nestlé Deutschland AG, Frankfurt am Main, Deutschland) pro Liter, um eine ausreichende Distension des Darmes zu erzeugen. Kurz vor der Untersuchung erhielten die Patienten 200 - $300 \mathrm{ml} \mathrm{NaCl} \mathrm{0,9 \%} \mathrm{als} \mathrm{rekta-}$ le Kontrastierung zur zusätzlichen Distension des Rektums [10, 11]. Zur Verstärkung der Darmdistension und zur Reduktion der Darmperistaltik während der Untersuchung erhielten alle Patienten $40 \mathrm{mg}$ Butyl-Scopolamin (Buscopan, Boehringer Ingelheim Pharma GmbH \& Co. KG, Ingelheim am Rhein, Deutschland) intravenös.

Zur Optimierung der Darstellung der entzündlichen Darmwandveränderungen erfolgte eine Kontrastverstärkung der T1-gewichteten Sequenzen durch eine intravenöse Applikation von Gd-DTPA (Magnevist, Schering AG, Berlin, Deutschland) in einer Dosierung von $0,1 \mathrm{mmol} / \mathrm{kg}$ Körpergewicht.

Die Untersuchung wurde an einem 1,5 Tesla-MR-Tomografen mit einer Gradientenfeldstärke von 20 mT/m durchgeführt (Symphony, Siemens Medical Systems, Erlangen, Deutschland). Die Patienten wurden in Rückenlage in zirkulären 4-elementigen Körperspulen untersucht. Zunächst wurden koronare T2 / T1-gewichtete-Sequenzen (TrueFISP, True Fast Imaging With Steady Precession, TR/TE 4,76/2,38 ms, Flip-Winkel 60, Matrix 256, FOV $450 \mathrm{~mm}$, Messzeit 19s) mit einer Schichtdicke von $5 \mathrm{~mm}$ und anschließend axiale T2-gewichtete Turbo-Spin-Echo-Sequenzen (HASTE, Half Fourier-Acquired Single Shot Turbo Spin Echo, TR/TE 1070/77 ms, Flip-Winkel 150, Matrix 256, FOV $400 \mathrm{~mm}$, atemgetriggert, Messzeit $109 \mathrm{~s}$ ) mit einer Schichtdicke von $8 \mathrm{~mm}$ angefertigt.

Zusätzlich wurden axiale fettgesättigte, T1-gewichtete Gradienten-Echo-Sequenzen (2D-FLASH, Fast Low Angle Shot, TR/TE 124/4,7 ms, Flip-Winkel 70, Matrix 256, FOV $380 \mathrm{~mm}$ ) mit einer Schichtdicke von $6 \mathrm{~mm}$ vor (Messzeit $72 \mathrm{~s}$ ) und nach Gd-DTPAApplikation (Messzeit 119 s) durchgeführt. Den Abschluss des Sequenzprotokolls bildete eine koronare fettgesättigte T1-gewichtete Gradienten-Echo-Sequenz (2D-FLASH, Fast Low Angle Shot, TR/TE 86/4,7 ms, Flip-Winkel $70^{\circ}$, Matrix 256, FOV $380 \mathrm{~mm}$, Messzeit $90 \mathrm{~s}$ ) mit $5 \mathrm{~mm}$ Schichtdicke. Die gesamte Untersuchungszeit belief sich auf durchschnittlich 26 min (Range: $23-$ $37 \mathrm{~min}$ ) inkl. der rektalen und intravenösen Kontrastmittelgabe. Sowohl die gewählten Sequenzen als auch die jeweils angegebenen Sequenzparameter entsprechen dem Routine-MRT-Protokoll an unserem Institut und sind über Jahre optimiert und etabliert, wobei jährlich mehr als 200 Patienten mit chronisch entzündlichen Darmerkrankungen mittels MRE untersucht werden.

\section{Auswertung der Daten}

Alle MR-Aufnahmen wurden von 4 erfahrenen Radiologen unabhängig voneinander subjektiv benotet. Die 4 Auswerter waren Fachärzte für Radiologie mit mindestens 3 Jahren Erfahrung in der Abdominaldiagnostik. Die Radiologen mussten für jede einzelne Sequenz subjektiv angeben, wie wichtig sie ihnen zur jeweiligen Diagnosefindung war. Die durchgeführten Sequenzen wurden diesbezüglich mit einer Ordinalskala entsprechend von Schulnoten bewertet ( 1 =sehr gut, 2 =gut, 3 =befriedigend, 4 = ausreichend, 5 = unbrauchbar). Alle Noten waren für alle Se- 
quenzen zur Vergabe möglich, verschiedene Sequenzen konnten auch mit der gleichen Note bewertet werden.

Bei insgesamt 29 Patienten (34,5\%) wurde unter Übereinstimmung aller 4 Radiologen keine entzündliche Aktivität diagnostiziert. Bei weiteren 20 Patienten (24\%) wurde übereinstimmend eine entzündliche Darmaktivität gefunden. In weiteren $35 \mathrm{~Pa}-$ tienten $(41,5 \%)$ wurde von mindestens einem, nicht jedoch von allen 4 Radiologen eine entzündliche Darmaktivität postuliert. Bei 12 Patienten (14\%) wurde unter Übereinstimmung aller 4 Radiologen eine singuläre Fistel oder eine komplexes Fistelsystem gefunden (jeweils perianal oder interenterisch).

\section{Statistische Methoden}

Für die Durchführung der statistischen Tests wurde das Programm SPSS in der Version 16.0 (SPSS Inc., Chicago, IL, USA) für Windows (Microsoft, Redmond, WA, USA) verwendet.

Die Rangfolge der MRT-Sequenzen, die sich aus der Benotung der 4 Radiologen ergab, wurde mit der Rangvarianz-Analyse nach Friedman auf ihre Signifikanz überprüft. Die Nullhypothese wurde bei einem $p$-Wert $<0,05$ signifikant und bei einem $p$-Wert $<0,01$ hochsignifikant verworfen. Da sich mit dem FriedmanTest aber nur eine Aussage treffen lässt, ob Rangunterschiede in der Stichprobe bestehen, nicht jedoch die Unterscheidungsrichtung oder Größe der Rangunterschiede feststellbar ist, wurde im Anschluss noch der jeweils erste Rang gegenüber allen nachfolgenden Rängen mit dem Vorzeichenrangtest nach Wilcoxon bestimmt. Auch hier erfolgt die Ablehnung der Nullhypothese bei $\mathrm{p}<0,05$ mit signifikant und bei $\mathrm{p}<0,01$ mit hochsignifikant.

\section{Ergebnisse}

$\nabla$

Nimmt man die subjektiven Benotungen der 4 Auswerter als Grundlage, ergibt sich als Reihenfolge der untersuchten Sequenzprotokolle als Erstes die HASTE (Durchschnittsnote 2,49), vor der kontrastmittelverstärkten 2D-FLASH koronar (Durchschnittsnote 2,60 ), erneut vor der TrueFISP (Durchschnittsnote 2,77) und vor der kontrastmittelverstärkten 2D-FLASH axial (Durchschnittsnote 2,85), zuletzt die 2D-FLASH axial mit einer Durchschnittsnote von nur 4,28 (๑ Tab. 1).

Der Rangvergabe liegt der im Friedman-Test ermittelte mittlere Rang zugrunde. Das Signifikanzniveau liegt bei $\mathrm{p}<0,005$.

Da der Friedman-Test nur generell eine Aussage trifft, ob zwischen den Bewertungen der einzelnen Sequenzen ein Unterschied besteht, wird im Folgenden noch das Ergebnis des nachgeschalteten Wilcoxon-Tests wiedergegeben; hier wurde geprüft, ob der Unterschied zwischen der Beurteilung der erstrangigen und allen nachrangigen Sequenzen signifikant ist.
Mit einem p-Wert $<0,05$ ist der Unterschied zwischen der erstplatzierten HASTE-Sequenz und der zweitplatzierten kontrastmittelverstärkten 2D-FLASH-Sequenz koronar signifikant. Alle weiteren Ergebnisse sind auf einem Signifikanzniveau von $\mathrm{p}<0,01$ als hochsignifikant zu werten.

Die nach den 4 Radiologen aufgeteilten jeweiligen Durchschnittsnoten der einzelnen Sequenzen können 0 Tab. 2 entnommen werden.

Bei getrennter Betrachtung der 4 beurteilenden Radiologen fällt auf, dass die Benotungen der Radiologen 1, 3 und 4 ein relativ einheitliches Bild ergeben: Die TrueFISP-Sequenz erhält neben dem HASTE-Protokoll die Bestnoten. Nur der Radiologe 2 weicht hiervon deutlich ab. Er vergibt für das TrueFISP-Protokoll die schlechtesten Benotungen. Wie deutlich sich die abweichende Benotung dieses Radiologen auswirkt, zeigt sich, wenn man die Gesamtübersicht nochmals nur für die Radiologen 1, 3 und 4 darstellt ( $\bullet$ Tab.3). Denn hierbei ergibt sich als Reihenfolge der untersuchten Sequenzprotokolle nun als Erstes die TrueFISP (korrigierte Durchschnittsnote 2,27) vor der HASTE (korrigierte Durchschnittsnote 2,54), vor der kontrastmittelverstärkten 2D-FLASH koronar (korrigierte Durchschnittsnote 2,70), erneut vor der kontrastmittelverstärkten 2DFLASH axial (korrigierte Durchschnittsnote 3,06) und zuletzt die 2D-FLASH axial (korrigierte Durchschnittsnote 4,43).

Beispielhaft zeigen die $\boldsymbol{\bullet}$ Abb. $\mathbf{1 - 5}$ den MRE-Untersuchungsgang eines 27-jährigen Patienten mit langjährig gesichertem MC, bei dem aufgrund der Symptomatik eines akuten Schubes eine MRE durchgeführt wurde. Es zeigte sich das Bild einer Ileitis terminalis und präterminalis.
Tab. 1 Rangfolge der MRT-Sequenzen bei der MRE für Radiologe 1 - 4. Anhand einer Ordinalskala (entsprechend Schulnoten) wurden die Sequenzen subjektiv nach ihrer Eignung zur Diagnosefindung bei MC bewertet ( $1=$ sehr gut, 2 = gut, 3 = befriedigend, 4 = ausreichend, 5 = unbrauchbar). Hierbei repräsentiert der mittlere Rang die errechnete Durchschnittsnote. ${ }^{1}$

\begin{tabular}{|c|c|c|}
\hline Rang & Sequenzprotokoll & $\begin{array}{l}\text { mittlerer Rang } \\
\text { (im Friedman-Test) }\end{array}$ \\
\hline 1. Rang & HASTE & 2,49 \\
\hline 2. Rang & 2D-FLASH koronar mit KM & 2,60 \\
\hline 3. Rang & TrueFISP & 2,77 \\
\hline 4. Rang & 2D-FLASH axial mit KM & 2,85 \\
\hline 5. Rang & 2D-FLASH axial & 4,28 \\
\hline
\end{tabular}

${ }^{1}$ mit KM= mit Gd-DTPA i. v.

Tab. 2 Bewertung der MRT-Sequenzen bei der MRE für Radiologe 1 - 4. Anhand einer Ordinalskala (entsprechend Schulnoten) wurden die Sequenzen subjektiv nach ihrer Eignung zur Diagnosefindung bei MC bewertet ( 1 = sehr gut, 2 = gut, 3 = befriedigend, $4=$ ausreichend, $5=$ unbrauchbar). Hierbei ergeben sich die angegebenen Durchschnittsnoten aus dem mittleren Rang im Friedman-Test. ${ }^{1}$

\begin{tabular}{|llll}
\hline Sequenz & Radiologe $\mathbf{1}$ & Radiologe $\mathbf{2}$ & Radiologe $\mathbf{3}$ \\
\hline TrueFISP & 2,76 & 4,30 & 2,34 \\
\hline 2D-FLASH koronar mit KM & 2,57 & 2,29 & 2,72 \\
\hline HASTE & 2,21 & 2,34 & 2,54 \\
\hline 2D-FLASH axial mit KM & 3,16 & 2,22 & 2,95 \\
\hline 2D-FLASH axial & 4,31 & 3,84 & 3,07 \\
\hline
\end{tabular}

1 mit KM= mit Gd-DTPA i. v. 


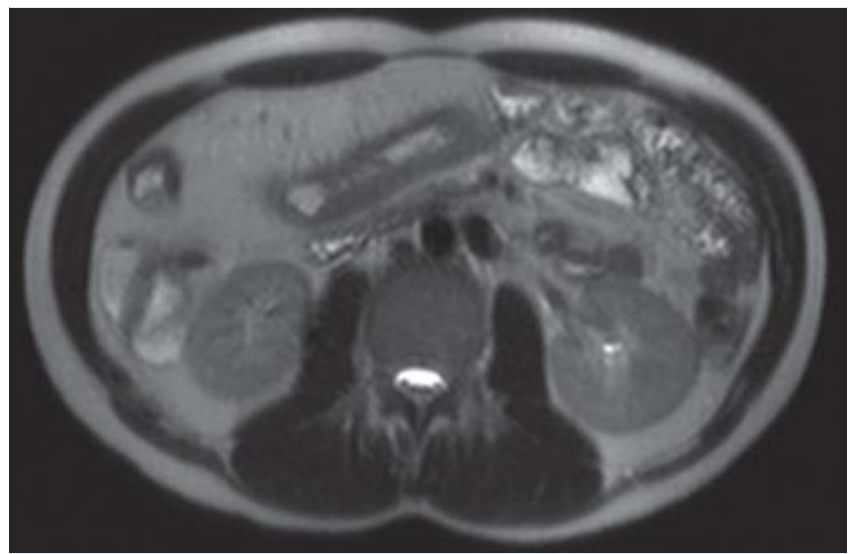

Abb. 1 -5 MRE eines 27-jährigen Patienten mit langjährigem MC mit der Symptomatik eines akuten Schubes mit diffusen Unterbauchschmerzen und blutigen Diarrhöen. Es zeigt sich eine lleitis terminalis und präterminalis mit langstreckiger Wandverdickung, langstreckigem KM-Enhancement der entzündlich veränderten Darmwand und umgebendem „creeping fat“. Konsekutiv kommt eine prästenotische Dilatation des vorangeschalteten lleums besonders in den koronaren Abbildungen zur Darstellung.

Abb. 1 Die axiale T2-gewichtete HASTE-Sequenz (TR/TE 1070/77 ms, FlipWinkel $150^{\circ}$, Matrix 256 , FOV $400 \mathrm{~mm}$ ) mit einer Schichtdicke von $8 \mathrm{~mm}$ zeigt sowohl das "creeping fat" als auch die langstreckige Darmwandverdickung im terminalen und präterminalen lleum. Subjektive Benotung (Radiologe1 -4): 2, 1, 2, 2.
Tab. 3 Rangfolge der MRT-Sequenzen bei der MRE für Radiologe 1, 3 und 4. Anhand einer Ordinalskala (entsprechend Schulnoten) wurden die Sequenzen subjektiv nach ihrer Eignung zur Diagnosefindung bei MC bewertet ( 1 = sehr gut, 2 = gut, 3 = befriedigend, 4 = ausreichend, 5 = unbrauchbar $)$. Hierbei repräsentiert der mittlere Rang die errechnete Durchschnittsnote. Für diese Berechnung erfolgte eine Elimination der Wertung von Radiologe 2, da dieser eine signifikant andere subjektive Bewertung der Sequenzen vorgenommen hatte. ${ }^{1}$

\begin{tabular}{|c|c|c|}
\hline Rang & Sequenzprotokoll & $\begin{array}{l}\text { Mittlerer Rang } \\
\text { (im Friedman-Test) }\end{array}$ \\
\hline 1. Rang & TrueFISP & 2,27 \\
\hline 2. Rang & HASTE & 2,54 \\
\hline 3. Rang & 2D-FLASH koronar mit KM & 2,70 \\
\hline 4. Rang & 2D-FLASH axial mit KM & 3,06 \\
\hline 5. Rang & 2D-FLASH axial & 4,43 \\
\hline
\end{tabular}

1 mit KM $=$ mit Gd-DTPA i. v.

\section{Diskussion}

\section{$\nabla$}

Bei Auswertung der aus den subjektiv vergebenen Schulnoten errechneten Gesamtränge aller 4 beurteilenden Radiologen ergibt sich - wie bereits gezeigt - als Reihenfolge die HASTE (Durchschnittsnote 2,49), vor der kontrastmittelverstärkten 2D-FLASH koronar (Durchschnittsnote 2,60), erneut vor der TrueFISP (Durchschnittsnote 2,77) und vor der kontrastmittelverstärkten 2D-FLASH axial (Durchschnittsnote 2,85), zuletzt die 2D-FLASH axial mit einer Durchschnittsnote von nur 4,28 ( $\bullet$ Tab. 1).

Eliminiert man aber den Radiologen 2 aus der Auswertung (er vergibt ja für das TrueFISP-Protokoll die schlechtesten Benotungen), ergibt sich eine neue Reihenfolge ( $\bullet$ Tab.3), nämlich die TrueFISP (korrigierte Durchschnittsnote 2,27) vor der HASTE (korrigierte Durchschnittsnote 2,54), vor der kontrastmittelver-

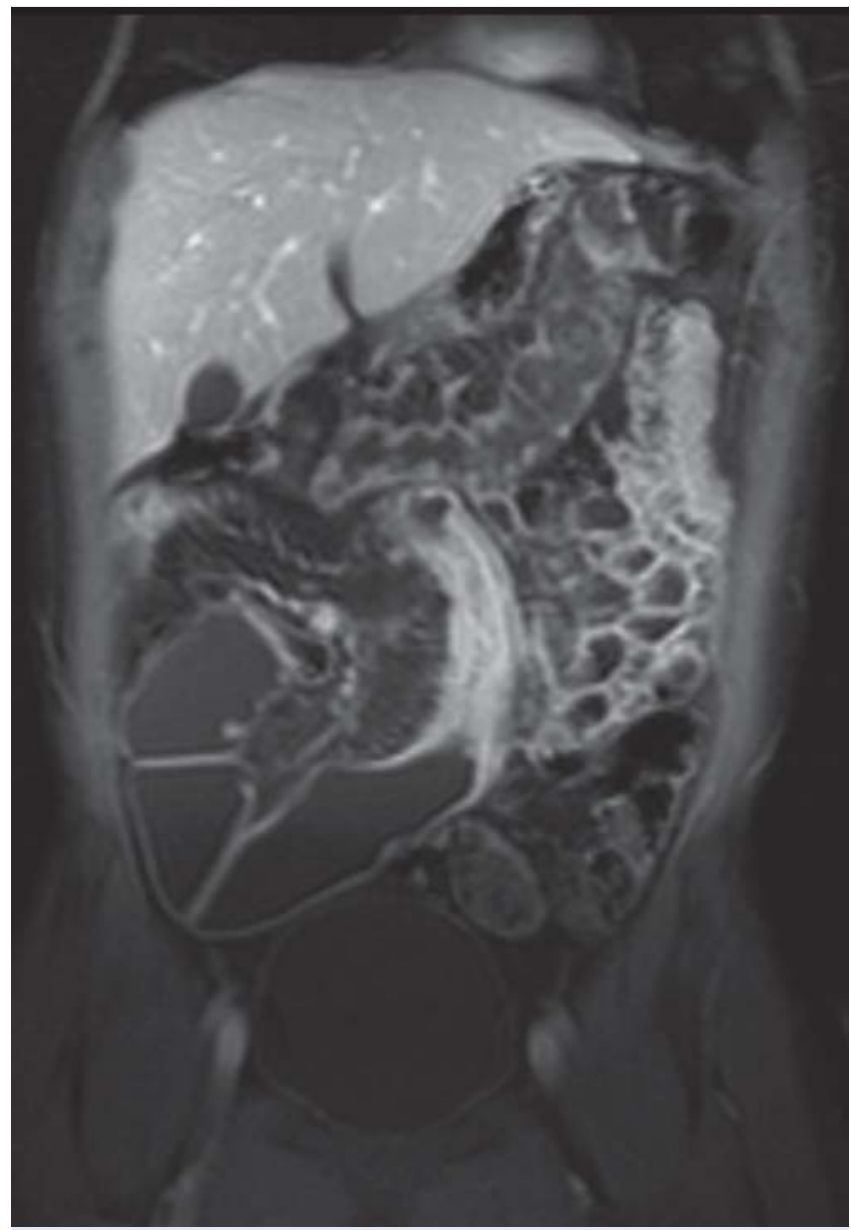

Abb. 2 Die koronare fettgesättigte 2D-FLASH (TR/TE 86/4,7 ms, FlipWinkel $70^{\circ}$, Matrix 256, FOV $380 \mathrm{~mm}$ ) mit $5 \mathrm{~mm}$ Schichtdicke nach GdApplikation zeigt sowohl das „creeping fat" als auch die langstreckige Darmwandverdickung im terminalen und präterminalen lleum mit konsekutivem KM-Enhancement. Subjektive Benotung (Radiologe1 -4): 2, 2, $1,2$.

stärkten 2D-FLASH koronar (korrigierte Durchschnittsnote 2,70), erneut vor der kontrastmittelverstärkten 2D-FLASH axial (korrigierte Durchschnittsnote 3,06) und zuletzt die 2D-FLASH axial mit einer korrigierten Durchschnittsnote von 4,43. Die TrueFISPSequenz erhält hier eine deutliche Aufwertung und läge somit vor dem in der Gesamtauswertung führenden HASTE-Protokoll. Die hier gefundenen Bewertungen stimmen auch mit den in der aktuellen Literatur zu findenden Aussagen überein [12, 13]. Die HASTE- und TrueFISP-Sequenz eignen sich unterschiedlich gut zur Diagnostik verschiedener MC-Symptome: So sind HASTEAufnahmen zur Diagnostik von oberflächlichen Erosionen und Wandverdickungen besser geeignet, TrueFISP-Aufnahmen (insbesondere durch das sogenannte Black-border-Phänomen) haben einen Vorteil bei der Darstellung von Wandulzerationen und mesenterialen Pathologien [12, 14].

Zur Etablierung eines optimalen Untersuchungsprotokolls zur Diagnostik bei MC müssen vielfältige Aspekte betrachtet werden. Zum einen wünscht sich der klinische Radiologe möglichst hochauflösende Sequenzen mit zueinander komplementären Informationen, zum anderen darf die Untersuchungszeit ein vertretbares Maß nicht überschreiten.

Die axiale HASTE- und die koronare TrueFISP-Sequenz wurden in den meisten Fällen subjektiv als gute bis sehr gute Sequenzen be- 


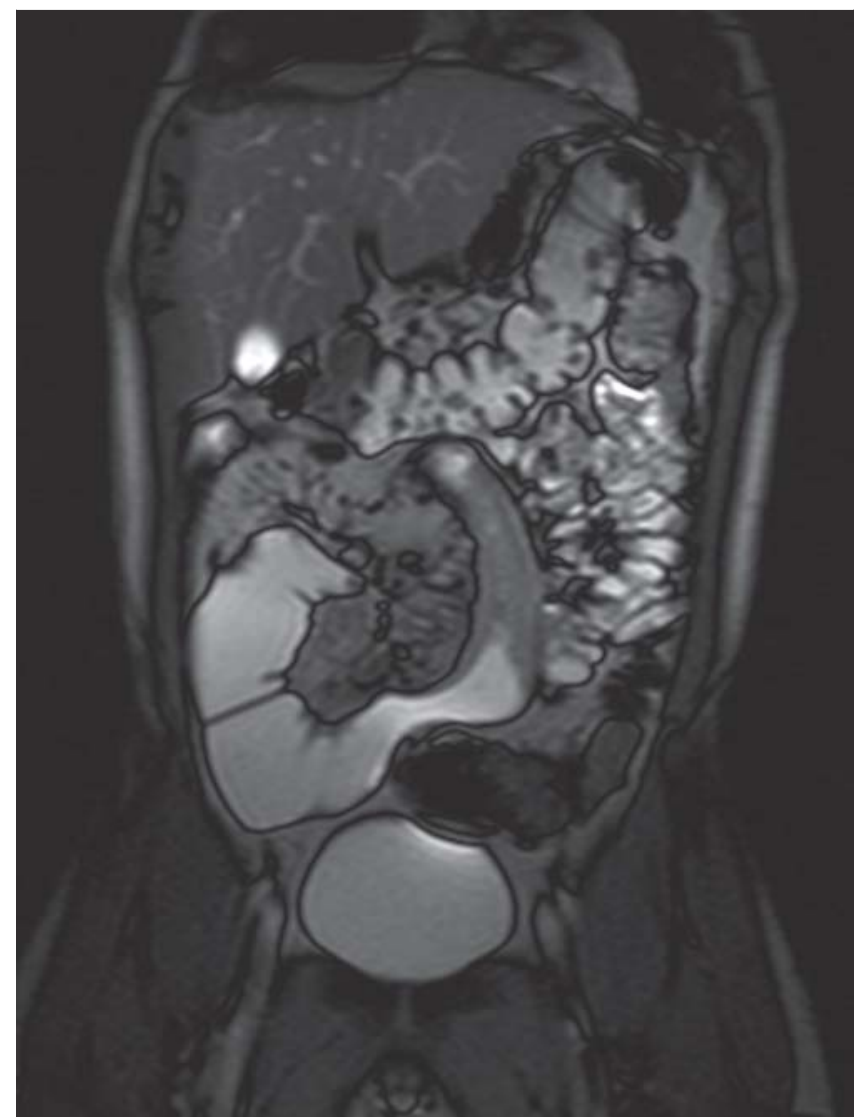

Abb. 3 Die koronare TrueFISP (TR/TE 4,76/2,38 ms, Flip-Winkel 60, Matrix 256, FOV $450 \mathrm{~mm}$ ) mit einer Schichtdicke von $5 \mathrm{~mm}$ zeigt sowohl das „creeping fat“ als auch die langstreckige Darmwandverdickung im terminalen und präterminalen lleum. Subjektive Benotung (Radiologe 1-4): 2, 3, 2, 2.

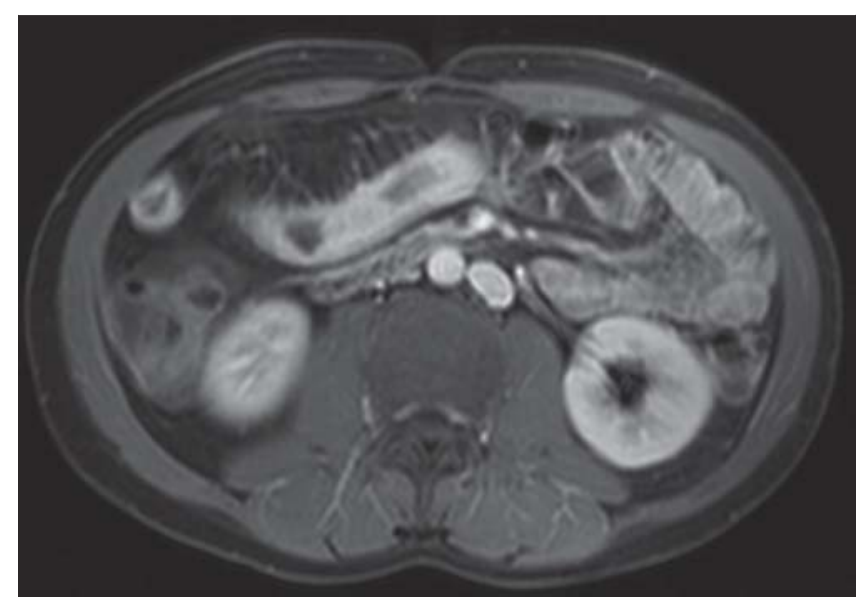

Abb. 4 Die axiale fettgesättigte 2D-FLASH-Sequenz (TR/TE 124/4,7 ms, Flip-Winkel $70^{\circ}$, Matrix 256, FOV $380 \mathrm{~mm}$ ) mit einer Schichtdicke von $6 \mathrm{~mm}$ nach Gd-DTPA-Applikation zeigt sowohl das „creeping fat" als auch die langstreckige Darmwandverdickung im terminalen und präterminalen lleum mit konsekutivem KM-Enhancement. Subjektive Benotung (Radiologe 1-4): 2, 1, 3, 2.

wertet, da sie den Auswertern eine stabile Bildgebung mit wenig Bewegungsartefakten lieferten. So konnte, basierend auf diesen Sequenzen, eine subjektiv sichere Aussage zur Darmwandverdi-

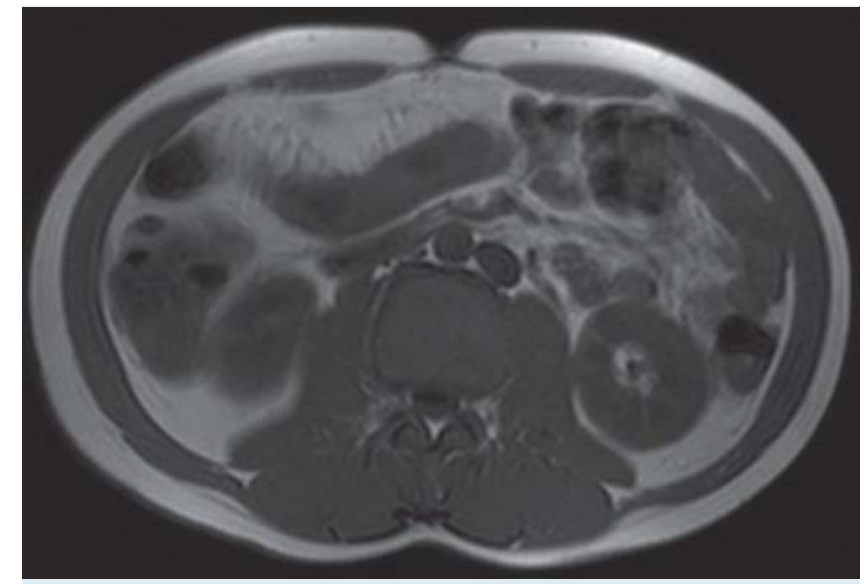

Abb.5 Die axiale fettgesättigte 2D-FLASH-Sequenz (TR/TE 124/4,7 ms, Flip-Winkel $70^{\circ}$, Matrix 256, FOV $380 \mathrm{~mm}$ ) mit einer Schichtdicke von $6 \mathrm{~mm}$ vor Gd-DTPA-Applikation zeigt sowohl das "creeping fat" als auch die langstreckige Darmwandverdickung im terminalen und präterminalen lleum. Subjektive Benotung (Radiologe 1-4): 3, 3, 2, 3.

ckungen gemacht werden. Durch die stabile T2-gewichtete Darstellung der HASTE-Sequenz war darüber hinaus eine Beurteilung eines Darmwandödems als Kriterium einer aktiven Entzündung möglich. Zur Beurteilung von vergrößerten mesenterialen Lymphknoten und zur übersichtlichen Darstellung der viszeralen Anatomie hinsichtlich potenzieller Stenosen wurden von den Auswertern vor allem die koronar akquirierten Sequenzen bevorzugt. Durch die übersichtlichere Darstellung der komplexen Darmanatomie in koronarer Schichtführung wurden auch innerhalb der kontrastmittelunterstützten Gradientenecho-Sequenzen die koronare T1-gewichtete FLASH-2D-Sequenz bevorzugt. Auch diese Sequenz hat sich in unserer Studie in Übereinstimmung mit der Literatur als wichtige MRT-Sequenz zur Beurteilung von Entzündungen und Fisteln herausgestellt [10, 15 - 17].

Die in dieser Studie gefundene Rangvergabe für die untersuchten Sequenzen zeigt zum einen, dass die koronare TrueFISP-Sequenz einen guten Überblick über pathologische Veränderungen bieten kann, zum anderen, dass gerade die T2-gewichtete HASTE-Sequenz durch die Darstellung von Ödemen wertvolle Informationen über pathologische Veränderungen bei MC liefert, da sie eine relativ artefaktarme Untersuchungsmethode darstellt, die die wesentlichen Veränderungen gut abbildet. In vielen Fällen scheinen diese Sequenzen ohne intravenöse Kontrastierung durch ihre schnelle Durchführbarkeit und Stabilität in der Bildqualität den radiologischen Auswertern subjektiv die wichtigsten und besten Sequenzen zur Diagnosefindung zu sein. Konsequent zeigt sich bei allen Auswertern, dass die native Gradienten-Echo-Sequenz subjektiv den geringsten Anteil zur Diagnoseentscheidung in der MRE beim MC beiträgt. Dies entspricht der eigenen Erfahrung, wobei diese native Sequenz noch häufig lediglich für Studienauswertungen zur Beurteilung der späteren Kontrastmittelaufnahme als Baseline-Untersuchung zusätzlich durchgeführt wird.

Stärken dieser Studie sind das prospektive Design mit 4 Untersuchern, welches versucht, den subjektiven Einfluss auf die Interpretierbarkeit von Bilddaten korrekt abzubilden.

An Schwächen hingegen wäre einerseits der fehlende Goldstandard zu nennen, andererseits zeigte sich, dass ein einzelner Untersucher eine signifikant abweichende Präferenzenliste im Vergleich zu den anderen Untersuchern angab und bereits das Abweichen eines Einzelnen die Rangreihenfolge abänderte. Andererseits kann 
als Schwäche gelten, dass lediglich eine subjektive Bewertung basierend auf einer Schulnotenskala als Grundlage der Etablierung einer Sequenzrangfolge diente und dass keine objektiven Kriterien in die Auswertung mit einbezogen wurden. Ferner wurden die mittlerweile zunehmend eingesetzten T1-gewichteten 3D-Gradienten-Echo-Sequenzen (z.B. 3D-FLASH, VIBE) nicht evaluiert. Diese spielen aktuell eine gesteigerte Rolle in der MRT-Diagnostik im Allgemeinen, der Zugewinn an Information jedoch bleibt umstritten und muss sich im klinischen Alltag erst noch beweisen. In aktuellen Studien sind 3D-FLASH-Sequenzen sogar zumeist den TrueFISP- oder HASTE-Sequenzen unterlegen $[18,19]$. Außer Acht blieben ebenso motilitätsabbildende Sequenzen („cine-MRT“), welche bisweilen einen diagnostischen Zugewinn zum RoutineUntersuchungsgang bei Patienten mit MC mittels MRT liefern, wie Froehlich et al. zeigen konnten [20].

Die MRT wird auch in Zukunft die Methode der Wahl zur Verlaufskontrolle und auch zur Komplementierung der Erstdiagnostik bei MC sein. Umso wichtiger ist es, ein optimiertes Sequenzprotokoll zu entwerfen, welches optimale Diagnosesicherheit mit vertretbarem zeitlichem Aufwand kombiniert. Die hier vorliegende Studie möchte zu diesem Ziel einen Beitrag leisten. Für die Zukunft wären doppelt geblindete Studiendesigns mit hohen Patienten- und auch hohen Reader-Zahlen wünschenswert, welche eine statistisch einwandfrei auszuwertende Rangfolge zwischen den vorliegenden Sequenzen ergeben und somit zur Entwicklung des idealen Untersuchungsprotokolls für Patienten mit MC in der MRE führen können.

Übereistimmend mit der aktuellen Literatur $[12-14,18,19]$ kann in der vorliegenden Studie gezeigt werden, dass die TrueFISP und die HASTE die bedeutendsten Sequenzen für die MRE-Diagnostik beim MC darstellen. Offensichtlich jedoch kommt den Gradienten-Echo-Sequenzen in koronarer und axialer Schichtführung nach Kontrastmittelgabe eine zunehmende Bedeutung zu, wohingegen die 2D-FLASH-Sequenzen ohne KM-Applikation nur eine untergeordnete Rolle zu spielen scheinen. Ferner muss sich noch zeigen, inwieweit diffusionsgewichtete Sequenzen in Zukunft eine Rolle in der Abdominaldiagnostik [21] und insbesondere bei der Detektion von entzündlichen Darmwandveränderungen bei Patienten spielen werden und ob diese gar mit den KM-gestützten Sequenzen konkurrieren können. Dazu gib es bereits vielversprechende Ergebnisse von Oto et al., die mittels diffusionsgewichteten Sequenzen in 18 von 19 Fällen die korrekte Diagnose von entzündlich veränderten Darmabschnitten zeigen konnten [22]. In weiterführenden Studien konnte die gleiche Forschergruppe um Oto et al. sogar die Überlegenheit von diffusionsgewichteten MRT-Sequenzen gegenüber KM-gestützten MR-Sequenzen für den Einsatz zur Detektion von aktivem MC im Dünndarm zeigen [23].

\section{Schlussfolgerungen}

\section{$\nabla$}

Basierend auf den subjektiven Auswertungen der MRE bei Patienten mit Morbus Crohn von 4 erfahrenen Radiologen stellen die T2 / T1-gewichteten Sequenzen der koronaren TrueFISP und die T2-gewichteten Sequenzen der axialen HASTE sehr wichtige und stabile MRT-Sequenzen dar, die entscheidend zur Diagnosefindung beitragen und mit Platz 1 und 3 im subjektiven Ranking gewertet wurden. Ergänzt werden sollte die MRE mit einer kontrastgestützten Gradienten-Echo-Sequenz vor allem in koronarer Orientierung, die ggf. noch mit einer zusätzlichen axialen Sequenz erweitert werden kann. Auf die native Gradienten-EchoSequenz kann am ehesten verzichtet werden.

\section{Literatur}

1 Schreyer AG, Seitz J, Feuerbach S et al. Modern imaging using computer tomography and magnetic resonance imaging for inflammatory bowel disease (IBD). Inflammatory bowel diseases 2004; 10: 45 - 54

2 Schreyer AG, Ludwig D, Koletzko S et al. Updated German S3-guideline regarding the diagnosis of Crohn's disease - implementation of radiological modalities. Fortschr Röntgenstr 2010; 182: 116-121

3 Hoffmann JC, Autschbach F, Bokemeyer B et al. Short version of the updated German S3 (level 3) guideline on diagnosis and treatment of Crohn's disease. Dtsch Med Wochenschr 2008; 133: 1924-1929

4 Van Assche G, Dignass A, Panes J et al. The second European evidencebased Consensus on the diagnosis and management of Crohn's disease: Definitions and diagnosis. J Crohns Colitis 2010; 4: 7-27

5 Masselli G, Casciani E, Polettini E et al. Comparison of MR enteroclysis with MR enterography and conventional enteroclysis in patients with Crohn's disease. Eur Radiol 2008; 18: 438 - 447

6 Schreyer AG, Geissler A, Albrich $\mathrm{H}$ et al. Abdominal MRI after enteroclysis or with oral contrast in patients with suspected or proven Crohn's disease. Clin Gastroenterol Hepatol 2004; 2: 491 - 497

7 Schill G, Iesalnieks I, Haimerl M et al. Assessment of disease behavior in Crohn's disease patients by MR-enterography. Inflamm Bowel Dis 2012, (in press)

8 Lee SS, Kim AY, Yang SK et al. Crohn disease of the small bowel: comparison of CT enterography, MR enterography, and small-bowel followthrough as diagnostic techniques. Radiology 2009; 251: 751 - 761

9 Schreyer AG, Friedrich C, Mrosek S et al. Cost Analysis of Contrast-Enhanced Cranial MRI at a German University Hospital. Fortschr Röntgenstr 2010; 182: 891-899

10 Schreyer AG, Scheibl K, Heiss P et al. MR colonography in inflammatory bowel disease. Abdom Imaging 2006; 31: $302-307$

11 Friedrich C, Fajfar A, Pawlik M et al. Magnetic resonance enterography with and without biphasic contrast agent enema compared to conventional ileocolonoscopy in patients with Crohn's disease. Inflammatory bowel diseases 2012; 18: $1842-1848$

12 Fidler J. MR imaging of the small bowel. Radiol Clin North Am 2007; 45: 317-331

13 Tolan DJ, Greenhalgh R, Zealley IA et al. MR enterographic manifestations of small bowel Crohn disease. Radiographics 2010; 30: 367-384

14 Parente F, Greco S, Molteni M et al. Oral contrast enhanced bowel ultrasonography in the assessment of small intestine Crohn's disease. A prospective comparison with conventional ultrasound, $\times$ ray studies, and ileocolonoscopy. Gut 2004; 53: $1652-1657$

15 Schreyer AG, Hoffstetter P, Daneschnejad M et al. Comparison of conventional abdominal CT with MR-enterography in patients with active Crohn's disease and acute abdominal pain. Academic radiology 2010; 17: $352-357$

16 Ajaj WM, Lauenstein TC, Pelster G et al. Magnetic resonance colonography for the detection of inflammatory diseases of the large bowel: quantifying the inflammatory activity. Gut 2005; 54: 257-263

17 Scheibl K, Schreyer AG, Kullmann F et al. Magnetic Resonance Imaging Gastrography: Evaluation of the Dark Lumen Technique Compared With Conventional Gastroscopy in Patients With Malignant Gastric Disease. Investigative radiology 2005; 40: 164-172

18 Gourtsoyiannis N, Papanikolaou N, Grammatikakis J et al. MR enteroclysis protocol optimization: comparison between 3D FLASH with fat saturation after intravenous gadolinium injection and true FISP sequences. Eur Radiol 2001; 11: 908-913

19 Masselli G, Vecchioli A, Gualdi GF. Crohn disease of the small bowel: MR enteroclysis versus conventional enteroclysis. Abdom Imaging 2006; 31: $400-409$

20 Froehlich JM, Waldherr C, Stoupis C et al. MR motility imaging in Crohn's disease improves lesion detection compared with standard MR imaging. Eur Radiol 2010; 20: 1945-1951

21 Schwartz CA, Haage P, Hohl C. Experimental early detection of acute mesenteric ischemia with functional MRI (DWI) and parallel imaging. Fortschr Röntgenstr 2012; 184: 520-526

22 Oto A, Zhu F, Kulkarni Ket al. Evaluation of diffusion-weighted MR imaging for detection of bowel inflammation in patients with Crohn's disease. Acad Radiol 2009; 16: 597-603

23 Oto A, Kayhan A, Williams JT et al. Active Crohn's disease in the small bowel: evaluation by diffusion weighted imaging and quantitative dynamic contrast enhanced MR imaging. J Magn Reson Imaging 2011; 33: $615-624$ 